das vorgeschrittene Stadium der Erkrankung allfs äusserste ersehöpftell Individuım operir'en müssen, das häuffig iiberhaupt einen scliweren Eingriff nicht mehr aushält. Man wül'de ferner', wenı man das Messer in die Hand nimmt, genall wissen, wo man das Hinder'niss in der Darmcirculation zu suchen hat, und würde also nicht, wie es jetzt noch in so vielen Fällen nötliig ist, gezwungen sein, die ganze Bauchhöhle zu durchsuchen, um den Sitz des Hindernisses festzustellen, ein Verfahren, bei clessen Ausfïhrung nieht so selten die gesunkenen Kräfte des Patienten völlig velsagen. Man würde schliesslich nicht mit den enormen Schwierigkeiten zu kämpfen liaben, welchen der bei längerer Dauel der Erkrankung vorhandene hochgradige Meteorismus einer schnellen Ausfiihrung der Operation bereitet, indem er den Zugang zur erkrankten Partie und die zur Beseitigung des Hiıdernisses nöthigen Eingriffe erschwert und selbst nach glücklicher Beseitigung des Hindernisses den Scliluss der Bauchwunde zu einem schwierigen und zeitraubenden Act macht.

Wenn nun auch iu den letzten Jahren die Specialdiagnose des Ileus manche Förderung erfahren hat, so namentlich dur'ch die schönen Arbeiten von v. Wahl und seinen Schülerı über dell Volvulus, so wird eine nach allen Richtungen vollkommene Diagnose des Ileus doch noch lange ein frommer Wunsch bleiben. Zum Glück sind nun bei einer Form des Ileus bereits alle Forderungen erfüllt, welche man in diagnostischer Beziehung stellen kann, und zwar gerade bei der Form des Ileus, welclie am allerhäufigsten ist und welche nach Leiclitenstern über $30 \%$ aller Ileusfälle umfassen soll, nämlich bei der Invagination. Das Symptomenbild der Invagination ist ein so scharf umschriebenes und setzt gleieh mit so voller Deutlichkeit ein, dass es in fast allen Fällen gelingt, bald die richtige Diagnose zu stellen. Den plötzlichen Beginn und die heftigen kolikartigen Schmerzen theilt die Invagination nit mauchen anderen Formen des Ileus, der Abgang von blutigem Schleim oder reinem Blut aus dem After macht, besonders wenı es sich um einen Patienten im kindliclıen Alter handelt, die Diagnose schon wahrscheinlicher, da er ausser bei Invagination nur noch bei Achselldrehung vorkommt. Sichergestellt aber wird die Diagnose durch del Nachweis eines wurstförmigeu Tumors, ein Symptom, welches, abgeseheu von einzelıen Fällen der relativ seltenen Dïnndarminvagination, stets bald nach Beginn del Erkrankung nachzuweisen ist. Ist der 'Tumor pel' rectum nachzuweisen, so ist seine Constatirung sehr einfach, aber auch von den Batchdecken aus gelingt der Nachweis ohne Schwierigkeit, wenn die Bauchdecken erschlafft sind. Sind sie aber, wie so liäufig, wegen der heftigen Schmerzen krampfhaft contrahirt, so wird man wenigstens an der Stelle der Invagination eine vermehrte Resistenz und eine leichte Dämpfung nachweisen können. Aber auch in diesen Fiallen schafft die Untersuchung in Narkose, die man dann nie verabsäumen soll, völlige Klarheit. Der Tumor, dell die illvaginilite Partie darstellt, ist in den meisten Fällen ein recht beträchtlicher und kanı meiner Ansicht nach bei der Untersuchung in Narkose dem "Untersucher niemals entgehen. Jedenfalls werden heute der diagnostischen Palpation des Unterleibes liäufig sehr viel schwierigere Aufgaben gestellt, als der Nachweis einer Invagination darstellt.

Wenn nun bei der Invagination die erste Voraussetzung eiuer

V. Aus der chirurgischen Abtheilung des israelitischen Krankenhauses in Hamburg.

\section{Zur operativen Behandlung der Darm- invagination.}

Von Oberarzt Dr. A. Alsberg.

Die operative Behandlung des Ileus ist in den letzten Jahren wiederholt Gegenstand der Discussion in den verschiedensteu $\mathrm{Ge}^{-}$ sellschaften des In- und Auslandes gewesen. Auch die Anhänger eines möglichst activen Eingreifens konıten uicht leugnen, dass die Erfolge der operativen Behandlung bis jetzt noch recht viel zu wïuschen übrig' lassen. Eine Verbessel'ung der Operationsresultate sei, das war gewöhnlich das Ergebniss der Discussion, in erster Linie zu erwarten von einer Vervollkommnung der Diagnose des Darmver'schlusses und dann von einer Vervollkomminung der operativen Technik. Und in der That, die schlechten Resultate, dje bisher mit der operativen Behandlung des Ileus erzielt worden sind, sind im wesentlichen begründet in der Unvollkommenheit unseres diagnostischen Könnens. Wenn es gelänge, in jedem Falle von Ileus gleich im Beginn der Erkrankung die Diagnose des Darmverschlusses im alloemeinen und die der speciellen Art des Darmverschlusses zu stellen, so würden damit die grössten Schwierigkeiten vermieden werden, die heute dem Operateur noch so oft entgegentreten und seine Bemühungen zu vergeblichen und undankbaren gestalten. Man würde zunächst nicht an einem durch 
invagination" veröffentlicht, in welcher das gesammte Material über Darminvagination von $1870-1892$ zusammengestellt und kritisch verwerthet ist. Es handelt sich im ganzen 1111725 Falle von Darminvagination. Dem Original, für dessen Ueberlassung ich Herrn Collegen Jessen zu Dank verpflichtet bin, entnehme ich folgende, die Resultate der Laparotomie bei Invagination betreffenden Zahlen:

Es wurden von 1870-1892 mit Laparotomie behandelt: Kinder unter 1 Jahr: 51, mit 9 Heiluugen. Kinder von 1-10 Jahren: 32 , mit 9 Heilungen. Ueber 10 Jahre: 55, mit 23 Heilungen, so dass es sich also im ganzen um 138 Operationen mit 41 Heilungen und 97 Todesfällen handelt.

Alle guten Resultate bei Kindern unter 10 Jahren wurden in Fällen erzielt, in welchen die Desinvagination gelang, alle 26 Fälle, in welchen die Desinvagination nicht gelang, starben, und zwar wurden von diesen 17 mit Darmresection, 7 mit Euterotomie behandelt. Von den Operationen bei Individuen über 10 Jahren wurdell 41 ausgeführt vor Ablauf des ersten Monats nach Beginn der Erkrankung. In diesen Fällell gelang 19 mal die Desinvagination, 12 Patienten wurden geheilt, von den 22 Patienten, bei welchen die Desinvagination nicht gelang, wurden nur 3 gerettet, und zwar wurde 14 mal die Darmresection ausgeführt mit 1 Heilung, 6 mal die Enterotomie mit 1 Heilung, 2 mal wurde die gangränöse Darmpartie in der Bauchwunde befestigt nit 1 Heilung.

Bei 14 nach Ablauf eines Monats Operirten wurden 8 geheilt, und zwar gelang in 6 Fällen die Desinvagination mit 4 Heilungen, in 8 Fällen wurde die Resection ausgeführt mit 4 Heilungen.

Aus der mitgetheilten Statistik geht hervor, dass die Resultate der operativen Behandlung der Invagination noch keine glänzenden sind.

Wenn auch manchem Patienten das Leben gerettet wurde, der ohne Operation sicher verloren gewesen wäre, so ist doch andererseits die Zahl derer, die nach der Operation starben, noch eine erschreckend hohe und eigentlich wohl geeignet, vor der Operation abzuschrecken. Abcr ist die hohe Mortalitätsziffer der Operation als solcher zur Last zu legen? Ich glaube nicht. Die Laparotomie bei Invagination ist, wenn sie von sachkundiger Hand in einem frühen Stadium ausgeführt wird, keine schwere Operation. Die exacte Diagnose des Sitzes der Invagination ist, wie oben ausgeführt wurde, in allen Fällen vor der Operation durch eine genaue Untersuchung zu stellen, man weiss daher nach Eröffnung der Bauchhöhle gleich, wo man die erkrankte Stelle zu suchen hat. Erheblichere Grade von Meteorismus sind bei der Invagination in einem früheren Stadium der Erkrankung fast nie vorhandeu. Die Beseitigung des Hindernisses, die Desinvagination, gelingt bei frühzeitig ausgeführter Operation in vielen Fällen sehr leicht. Da nun schliesslich auch im Beginn der Erkrankung, abgesehen von den peracuten Fällen bei klcinen Kindern, ein Collaps nicht besteht, so ist in der That nicht einzusehen, warum nicht die frühzeitig ausgeführte Laparotomie bei Invagination zu einem guten Resultate fuihren sollte. Wenn diese Erwägung im allgemeinen eine richtige ist, so ist für die schlechten Resultate, die bisher mit der Laparotomie erzielt wurden, im wesentlichen die zu späte Ausführung der Operation verantwortlich zu machen. Diese wird in vielen Fällen wohl dadurch veranlasst, dass die Diagnose zu spät gestellt wird, in einer grossen Reihe von Fällen aber dadurch, dass nach richtig gestellter Diagnose zu viel Zeit mit der Ausführung der sonstigen therapcutischen Maassnahmen verloren wird. Es besteht da ein Circulus vitiosus. Die Erfolge der Laparotomie sind bis jetzt nicht günstig, deshalb wird erst, wenn trotz Anwendung aller sonstigen Therapie der Zustand imme schlechter geworden ist, zur Laparotomie geschritten, weil aber dann gewöhnlich zu spät eingeschritten wird, ist das Resultat wieder ein schlechtes. Ich will damit nicht sagen, dass in jeden Falle von Invagination gleich die Laparotomie ausgefïhrt worden soll. Im Gegentheil, man soll in jedem frischen Fall die weniger cingreifenden Maassnahmen, die ja zweifellos in vielen Fällen eine Heilung horbeiführen, zunächst anwenden. Die hohen Wassereingiessungen, cventuell in Narkose, sind sicher ein sehr wirksames und, da der Druck beliebig regulirt werden kann, ein relativ ungefährliches Mittel. Statt der Wassereingiessung die Lufteiublasung vorzunehmen, dürfte sich nicht empfehlen, der Druck lässt sich dabei nicht genau reguliren, und Darmperforation kann die Folge zul encrgisch ausgeführter Lufteinblasung sein. $\mathrm{Knaggs}^{1}$ ) hat acht derartige Fälle mitgetheilt. Die Massage, die in jüngster Zeit erst wieder von $\mathrm{Harder}^{2}$ ) an der Hand von zwei günstig verlaufenen Fällen empfohlen wurde und von deren erfolgreicher Anwendung auch Wichmann in mehreren thcils von ihm, theils von Studsgaard behandelten Fällen berichtet, ist sicher cine ratio-

1) Lancet 1887, June 4, ref. Virchow-Hirsch, Jahresberichte.

2) Berl. klin. Wochenschr. 1893, No. 49 . nelle und in manchen Fällen zum Ziele führende Methode. Aber nur in eincm Bruchtheil der Fälle wird man anf diesem Wege zum Ziele gclangen, in viclen Fällen werden die Bemühungen crfolglos bleiben. Daun soll man abcr, das kanm mciner Ansicht nach nicht stark genug betont werden, nicht kostbare Stunden und Tage mit immer erneuten Versuchen verlieren, sondern man soll baldigst zur Ausführung der Laparotomie schreiten.

Die Invagination lässt sich galız gut mit der Brucheinklemmung vergleichen. Auch bei dieser gelingt es in vielen Fällen, ohne blutige Operation, durch Taxis, eine Heilung herbeizufiihren, und der behandelnde Arzt wird daher in jedem frischen Falle erst die Taxis versuchen. Gelingt die Taxis aber nicht, so inacht er sich nach allgemein gültigen Anschauungen eines Kunstfehlers schuldig, wenn er den Patienten über Nacht liegen lässt, um am nächsten Tagc die Versuche der Taxis zu wiederholen; er wird vielmehr dafür Sorge tragen, dass die Einklemmung durch einen operativen Eingriff baldigst gehoben wird. Denn er weiss, dass jedes weitere Zuwarten den Zustand des Patienten verschlechtern kann. Ganz ebenso verhält es sich bei der Invagination. Auch hier kann il jedem Falle über Nacht eine solche Vcrschlimmerung des Zustandes eintreten, dass die Operation, die heute noch die günstigsten Chancen bieten würde, morgen vielleicht schon aussichtslos erscheint. Man wird mir nun erwidern, dass in einer Reihe von Fällen doch durch immer wieder ausgeführte Wassereingiessungen oder Lufteinblasungen noch Heilung erzielt worden sei. Das ist richtig, doch ist die Zahl der auf diese Weise erzielten Erfolge sicher eine geringe gegen die grosse Zahl von Fällen, in welchen bei dieser Behandlung der Exitus eintrat, und man kann in keinem einzigen Falle vorhersagen, dass man in ihm durch wiederholte Anwendung der conservativen Maassnahmen zum Ziel gelangen wird. Dagegen lässt sich behaupten, dass in den Fällen, in welchen die Desinvagination ohne Operation später doch noch gelingt, aucl die Laparotomie verhältnissmässig günstige Chancen bietet, da man ja sicher in diesen Fällen auch bei der Laparotomie die Desinvagination leicht ausführen kann.

Es muss daher dic Forderung aufgestellt werden, dass in jcdem Fall von acuter Invagination, wenn die conservativen Maassnahmen ohne Erfolg angewendet sind, so bald als möglich die Laparotomie ausgeführt werden soll. Senn ${ }^{1}$ ) empfiehlt, in Narkose den Versuch zu machen, durch Lufteinblasung dic Invagination zu reponiren, und wenn dies nicht gelingt, direkt die Laparotomie anzuschliessen. Das wird sich jedoch nur im Hospital, wo alle Hülfsmittel gleich zur Hand sind, ausführen lassen.

Wenn nach diesen Prinzipion vorgegangen wird, und der praktische Arzt die Invagination ebenso wie den eingeklemmten Brucl als nicht zur Domäne der inneren Medicin gehörig ansieht und sie daher baldigst dem Chirurgen überweist, dann werden wir, das ist meine feste Ueberzeugung, bald eine bedeutende Verbesscrung der Opcrationsresultate zu verzeichnen haben.

Leider nehmen die gebräuchlichen Handbücher, namentlich der inneren Medicin, die für das Handeln des praktischen Arztes maassgebend sind, häufig noch einen wesentlich anderen Standpunkt ein. Da heisst es: In verzweifelten Fällen bleibt noch die Laparotomie übrig, und ähnlich. Selbst Braun, der sonst ja sehr warm für die frühzeitige Laparotomie eintritt, macht die Einschränkung, dass dieselbe auszufuihren sei "bei allen Invaginationen mit heftigen Eillklemmungserscheinungen ". Schon Meinhard Schmidt ${ }^{2}$ ) hat darauf hingewiesen, dass diese Beschränkung der Indication zur Operation nicht richtig ist, sondern dass wir gerade dahin strebel müssen zu operiren, ehe heftige Einklemmungserscheinungen eintreten. Denn ist erst völlige Obstipation und Meteorismus eingetreten, so operiren wir unter viel schwierigeren Verhältnissen, und alle die verhältnissmässig günstigen Chancen, die die Invagination gegenüber anderen Arten von innerer Einklemmung bietet, sind verloren.

Schmidt sagt am Schlusse seines Aufsatzes: Vielleicht verhilft eine kleine Reihe cclatanter günstiger Erfolge der operativen Therapic der Invagination schneller zum Siege, als wir es erwarten. Als cinen klcinen Beitrag in diesem Sinn möchte ich mir crlaubcn, aus meiner Abthcilung drei in den letzten drei Jahren beobachteto Fälle von Invagination mitzutheilen, welche dur'ch die Laparotomie und Desinvagination geheilt wurden.

Fall 1. Heinrich K., 5 Jahre alt, aufgenommen am 10. Januar 1891. Der Knabe erhielt vor acht Tagen einen Stoss gegen den Unterleib und erkrankte bald nachher mit heftigen Leibschmerzen. Die Schmerzen traten anfallsweise auf und waren so heftio, dass der Junge laut schrie und kaum zu halten war. Bald stellten sich Durchfälle ein, denen blutiger Schlein beigemischt war. Trotz grosser Dosen Opium trat eine Besserung vicht ein. Der behandelnde Arzt, D1. Adam, constatirte einen 'Tumor in der Nabelgegend und schickte den Patienten ins Krankenhaus. Abge- 
magerter, mässig kräftig g'ebanter Knabe. Tenıperatur mässig crhölıt (38,2), Puls etwas beschlemnigt. Gesichtsausdruek ängstlielı. Patient liegt meist nit gegen den Bauch gezogenen Beinen da. Klagt iiber bestandige Sehnerzen im Leibe, in Intervallen werden die Schmerzen heftiger. der Junge schrcit dann lant anf und krümmt sich vor Schmerzen. Das $\mathrm{Ab}$ domen ist nicht wesentlich aufgetrieben, die Bauchdecken sind straff gespannt. so dass eine genauc Palpation nicht möglich ist, doch fiilltt man oberhalb des Nabels eine vermehrte Resistenz. In Narkose fïlllt man ganz dentlich in den sonst ganz weichen und nicht anfgetricbenen Leibe im Verlauf des Colon transversum cinen ungefähr $10 \mathrm{~cm}$ langen wurstförmigen Tumor, der ungefälır zurr Hälfte rechts und zur Hälfte links von der Mittellinie liegt. Der Stnlllgang ist diarrhoisch, von gelber Farbe und anshaftem Gestank. Es finden sich in ilm reichliche butig gefïrbt Schleimfetzen. Nach diesem Befund war die Diagnose einer Invagination sichergestellt. Es wurde Opium innerlich weitergegeben, dabci wurden hohe Eingiessungen gemacht. Nach der Eingiessinng schien der Tumor zunächst kleiner, doch ergab sich bei genauerer Untersnchung, dass derselbe nur mehr nach rechts verschoben war und theilweise unter dem Rippenbogen versehwand. Auch an den beiden nächsten Tagen wurdeu die Eingiessungen angewendet, olne wesentlichen Erfolg. Die Schmerzanfälle wurden zwar etwas seltener, doch bostand der Tumor in unveräinderter Grösse fort. Deshalb wurde am 14. Januar zur Laparotomic geschirtten. Ausgiebiger Bauchsclnitt in der Linea alba, den Nabel links umkreisend. Es gelang leicht, die invaginirte Darmpartie zn finden und aus der Banchhöhle herauszuheben. Es fand sich, dass der unterste Theil des Ileum und das Coecum in das Colon ascendens invaginirt waren. Die Desinvagination gelang leicht durch passenden Zug und Druck, der Darm zeigte keime wesentlichen Eruährungsstörungen, nur an einer Stelle des Colon ascendens fand sich cin grösseres Blutextravasat. Schluss der Banchwunde. Der Verlanf nach der sehr rasch vollendeten Operation war ein ganz ungestörter, Temperatursteigerung trat nicht ein, der Stullgang enthiclt in den nielssten Tagen reichlich Eiter, doch kein Blut mehr. Der Knabe erholte sich rasch und konnte am 14. Februar geheilt entlassen werden. Irgend welche Störungen von Seiten des Darmeanals haben sich bei ihm nicht wieder eingestellt.

Fall 2. Franz M., 11 Jahre alt, aufgenommen am 19. Juni 1893, Abends. Der Knabe erkrankte an vorhergehenden Abend nach bestem Wohlbefinden plötzlich mit sehr heftigen, anfallsweise auftretenden Leibschmerzen. Tagrs zuvor war er von einem Mitschüler gegen den Banch gestossen worden. Der behandelnde Arzt, Dr. Zacharias, constatirte einen Thumor im Leibe, stellte die Diagnose anf Invagination und sehiekte den Knaben ins Krankenhaus zur eventuellen Operation. Patient macht einen leidenden Eindruek, Augen halonirt, Faeies abdoninalis. Beine an den Leib gezogen, Atlımung etwas beschleunigt. Puls 120, mittelkräftig. Von Zeit zn Zeit sehr heftige Sehmerzen im Leibe, auch in der Zwischenzeit ist der Leib empfindlich. Leib nieht aufgetricben, rechts von Nabel fïhl man deutlich einen wurstförmigen Tumor von zienılich derber Consistenz, der in toto verschieblich ist und tynıpanitisehen Schall giebt. Der Stuhlgang entliilt fast reines Blut. Kein Erbrechen. Die Diagnose einer Invagination stand fest, es wurden Massage und Lufteinblasungen ohne Erfoly versucht, nur wanderte bei den letzteren der Tumor mehr nach rechts in die Gegend der Flexur. Opium. Am anderen Morgen Temperatur 37,4, Puls 120. Nachts nicht geschlafen, nicht gebrochen. Stuhlgang wieder blutig. Leib ziemlich stark aufgetrieben, in toto druckempfindlich. Laparotomie. Bauchschnitt zur Hälfte oberhalb, zur Hälfte unterhalb des Nabels gelegen. Nach der Eröffnung der Banchböhle entleert sich viel klare, gelbe Fliissigkcit. und es drängen sich stark injieirte, geblähte Diïndarmsehlingen in die Wunde. Nach rechts kommt man auf die invaginirte Darmpartie. Die Invagination ist nicht leicht zul lösen, da das Intussusceptum schon stark eingeklemmt ist und ausserdem die aufgeblähten Dïnndarmschlingen sehr hinderlich sind. Doch gelang es allmählich. durch Zug am Dünndarm und Druek auf den Tumor die Invagination zu lösen. Das Intussuseeptum wird gebildet vom Anfangstheil des Colon ascendens, vom Coeeum und dem mitersten Theil des Ileum. Dio Invagination bildete einen wurstformigen vorderarmdicken Tumor von etwa $20 \mathrm{~cm}$ Lïnge. Die Darmwand zeigte sich besonders im Bereich des Coecum oddematös infiltrirt. an zwei Stellen war die Serosa durch dic Manipulationen eingerissen, doch nur unbedeutend. Nach Reposition des I)armes wurde die Banchhöhle dureh dic Naht gesehlossen. Der Verlauf war nicht so glatt wie im ersten Falle. Patient fieberte die ersten Tage. bis 39,0, der Puls war klein und frequent, 120-140, er klagte viel niber Lcibschmerzen, am zweiten Tage trat ein heftiger Kolikanfall ein, der sich melirfach wiederholte. Am dritten Tage erbrach Patient massenhaft gallige Flïssigkeit, danach wurde das Befinden besser. Die Leibsehmerzen traten nur noeh mässig' auf, die 'T'emperatır fiel zur Norm ab, der Puls wurde alluällich langsanıer und krïftiger. Die Reconvalescenz machte dann rasche Fortschritte, so dass Patient am 8. Juli, also am 19. Tage nach der Operation geheilt entlassen werden konnte.

Fall 3, Heinrich P. 18 Jahro alt, aufmenommen am 2. März 1895 Der Patient, der vor seiner Erkrankung sehr kräftig und gut genäln't war, erkrankte 16 Tage vor der Aufnahme plötzlieh mit heftigen Leibschmerzen und Kollern im Leibe, so dass er sich zu Bett legen nusste. Die ersten zwei Tage erfolgte kcin Stuhlgang, an dritten Tage breiiger, mit Blut vermischter Stuhlgang, sehr ubelriechend, zwei Stechbecken voll; gleichzeitig galliges Erbrechen mit Blutfasern untermischt. Die heftigen Kolikschmerzen dauerten an, nach drei Tagen erfolgte nach Ricinusül wieder blutiger Stuhl und Erbrechen. dann wieder drei Tage Verstopfung, bis anf Ricinusöl wieder blutiger Stılhlgang und Erbrechen erfolgte. Am nächsten T'age wurde ein Klystier von Stärke nit Opiumzusatz applieirt, daranf liessen die Schmerzen etwas nach, das Klystier blieb fünf Tago stehen, bis am Tage vor der Aufnahme sich wieder heftige Sehmerzen einstellten. Auf einen Wassereinlauf erfolgte dam erst gebundener Stuhl, daun ein Stechbecken voll Blut, zugleich trat rein blutiges, später grünliches Erbrechen cill. Das Befinden des Patienten hatte sich immer inchr verschlechtert. An 2. Miriz Mittags sah ich den Patienten zulerst in seiner Wolınung. Iclı fand einen sehr abgemagerten, hohläugigen Menschen von graucr Gesichtsfarbe. die Zunge war trocken, der Puls 120 von sehr geringer Spannung. Das Abdomen war mässig anfgetrieben, auf Druck ilberall, besonders rechts vom Nabel, empfindlich, hier war auch cine dentliche Resistenz zu fïlılen. In der rechten Weiche gedümpfter Sclall. Nach der Anamnese und dem Befund bei der Untersuchung hielt ich die Diagnose einer Invagination für im höchsten Grade wahrscheinlich und veranlasste die sehleunige Ueberführung des Patienten ins Krankenhans. Ieh sah dann den Patienten Abends im Krankenhaus wieder. Icl besehloss, ihn zunäehst in Narkose zu untersuchen und, falls die Untersnchung in Narkose dic Dingnose bestätigen würde, sofort zur. Laparotomie zu schreiten. Der Krïftczustand des Patienten sehien mir langes Experimontiren mit unsichcren Mittcln nicht zu gestatten, auch finrehtete ich bei dem langen Bestehen der Invagination und den hochgradigen Ernällrungsstörnngen, dic in den starken Blutungen ibren Ausdruck gefunder hatten, dass eine energisehe Anwendung von Wassereinläufen oder Lufteinblasungen schlimme Folgen haben, d. h. eventuell zur Darmperforation führen künnten. In Aethernarkose fühlte man rechts am Nabel cine wurstfơrnige, von links unten nach rechts oben sich erstrcekende, nach oben leicht eoneave Gesehwulst von mässig derber Consistenz. dic ziemlich gut verschieblich war und sich ganz ähnlich wie eine Wanderluiere anfühltc. Mit diesen Bcfund war die Diagnose einer Invagination gesichert, ich schritt daher zur Laparotomic. Bauchsehnitt in der Mammillarlinie vom Rippenbogen abwärts $8 \mathrm{~cm}$ lang. Aus der geơffneten Banchhöhle entlecrt sich reichlich blutig gefürbte Flïssigkeit. Das Netz wird nach oben geschoben, es drängen sich aufgetriebene, stark injicirte und mit punktförmigen Hämorrhagiecn durehsetzte Dïnndarmsehlingen in die Wunde und verlegen den Zngang zum 'Tumor. Sie werden daher allmählich ans dem Banch heransgezogen, dabei zeigt sich an einer Stelle eine deutliche Schnürfurche. Jetzt gelangt die eillgeführte Hand an eine resistentere Darmpartie, die herausgeholt wird. Es zeigt sich, dass es das Coecum ist, welches eigenthümlich veländert ist. Es ist in toto stark geröthet und ödematös gequollen, und sein blindes Ende ist so stark infiltrirt und verdickt, dass ich erst glaubte, es mit eincr Neubildıng des Darms zı thun zu haben. Die Schwellung ist aber eine so wciche, dass sie bequem mit dem Finger weggedrïekt werden kann, es handelt sich also nur um ein pralles Oedem. Dic eigentliche Kuppe des Coeeums ist etwas nach innen cingestïtzt und erscheint vollständig blutig infareirt. Nach diesem Befund kounte es keinem Zweifel unterliegen, dass wir es mit einer Invagination des Coecums und Ileums in das Colon ascendens zu thun hatten und dass die Invagination gleich zu Anfang der Operation mit dem Herausziehen der Dünndarmschlinge gelöst worden war. Nalıt der Banchwundo mit Scide. Puls am Ende der Operation 140. Kampherinjectionen, Cliampagner, starker Kaffee, später wegen heftiger Wundschmerzen 0,01 Morplinin subcutan. Der weitere Verlauf war dureh verschiedene Zwischenfälle gestört. Es trat eine doppelseitige Parotitis auf, ausserdem eine Anschwellung beider Sehultergelenke. Es trat an allen Stellen Rüekbildung ohne Eiterung ein. In den ersten acht Tagen war dic Temperatur leicht febril. Die Kolikschmerzen hörten mit der Operation auf, nach zwci Tagen erfolgte Stnhlgang, dem noch etwas Blut beigemischt war. Patient hatte sohr starken Durst, er trank sehr viel, durch zu reichliche Flüssigkeitsaufnalıme kam es fuinf Tage nach der Opcration zu heftigem Erbrechen, Singultus und Leibschmerzen, nach Einschränkung der Fliissigkeitszufuhr gaben sich aber bald alle Beschwerden. Noch an 12. Tage nach der Operation zeigte sich Blut im Stuhl. Nachdem Patient schon cine Reihe von Tagen leichte Fleischspcisen etc. verzelirt hatte, trat am 24. Tage nach der Operation wiederum Erbrechen und Leibschmerz ein, Patient wird daher wieder anf fliissige Diüt gesetzt und befand sich dabei, abgesehen von dem lebhaften Hungergefühl, völlig wohl. Doch erholte er sich nur sehr langsam und macht ganz den Eindruck eines Reconvalescenten von einem schweren Typhus. Am 5. April wurde er geheilt entlassen.

Ueberblicken wir noch einmal die drei Fälle, so handelte es sich in allen dreien um männliche Individuen im jugendlichen Alter. Das männliche Geschlecht ist belkanntlich bei der Invagination besonders bevorzugt. In zwei Fällen wurde ein kurz vorher stattgefundenes Trauma als Ursache der Erkrankung angegeben. In allen Fällen trat die Erkiankung plötzlich ein mit sehr heftigen Koliken, in den beiden crsten Fällen trat bald Stuhlgang ein, in dem dritten erst nach zweitägiger Verstopfung, in allen Fällen enthielt der erste Stuhlgang Blut. Erbrechell trat in dell ersten zwei Fällen nicht ein, im dritten jedoch gleichzeitig mit dem ersten Stuhlgang. Dem Erbrochenen war Blut beigemengt, auch im spiiteren Verlauf der Krankheit orfolgte noch einmal Erbrechen einer grösseren Menge von Blut. In allen drei Fällen war mit Sicherheit ein wurstförmiger Tumor nachzuweisen, in zwci Fällen allerdings nur nit Zuhülfenahme der Narkose, während man ohne Narkose nur eine schmerzhafte Resistenz fühlen konnte.

Die Operation selbst und der Verlauf nach derselben bieten in den drei Fällen grosse Verschiedenheiten. Am günstigsten lag der erste Fall. Obgleich bei der Operation schon zehu Tage seit Beginn der Erkrankung verflossen waren, waren die krankhaften Erscheinungen nur mässige, und der. Kräftezustand des Patienten cin guter. Bei der Operation war kein störender Meteorismus vorhanden, dic Desinvagination gelang leicht, der Darm zeigte an der eingestülpten Stelle keine wesentlichen Ernährungsstörungen. Dem 
entsprechend war der ganze Verlauf ein völlig glatter, ohne jeden stör'enden Zwischenfall. Im zweiten Falle war der' Verlauf ein stürmischerer; obgleich er noch innerhalb der ersten 48 Stunden nach Beginn der Frkrankung operirt wurde, war bereits ein, wenn auch mässiger Meteorismus vorhanden, dic Eı'nähr'lugsstöl'lungen im afficirten Darmtheil waren hochgradiger, wie man es auch aus den fast rein blutigen Entleerungen schliessen konnte. Die Desinvagination war schwieriger, die Reconvalescenz gestört durch Kolikanfälle, die wolıl auf eincr Parese des Darmes an der kranken Stelle beruhten. Doch erholte sich Patient rascl, weil der Gesammtorganismus durch die Erkrankıng noch niclıt schr geselıwächt war. Am schwersten war der di’tte Fall. Der Patient, der iı einem Zustand völliger Erschöpfung am 16. Tage nach der Fikrankung zur Operation kam, bot nur wenig Aussicht anf einen glïckliclien Ausgang der Operation. Die rcichlichen blutigen Fintleerungen sowic das blutige Erbrechen liessen anf tiefgreifende Veränderungen an der erkrankten Stelle sclıliessen, dabei nnachte der Patient entschieden einen Findruck von begiunender Sepsis. In der That fanden sich bei der Operation sehr hochgradige Veränderungen an der invaginirten Darmpartie; an der Stelle, die wir als Spitze des Intussusceptums betrachten müssen, war die Voränderung bis zur völligen blutigen Infarcirung des Darmes vorgeschritten, im Abdomon fand sich reichlich blutige Flüssigkeit. Trotzdem ging merkwürdiger Weise die Desinvagination sehr leicht von statten. Aber der Organismus vermochte die lokalen und allgemeinen Störungen, die durch die Krankheit entstanden warell, nur schwer zu überwinden. Infolge der ersteren latten wir mohrfach noch heftige Kolikanfällc mit Krbrechen zu beobachticn, sowie Blutabgang noch längcre Zeit nach der Operation, infolge der letzteren entstanden die Frkrankungen der Parotiden und der Schultergelenke, die wir doch sichır auf pathogene Substanzen zurückfïhren mïssen, die am Locus morbi in dic Blutbahn aufgenommen worden sind. So schritt die Reconvalescenz nur sehr langsam vor, und noch vier Wochen nach der Operation war der Patient sehr abgemagert und machte cinen leidenden Findruck.

Wir sehen also, dass in allen drei Fällen der operative Eingriff als solcher kein schwieriger war und dass die Gefahren, dencn die Patienten nach der Operation ausgesctzt waren, nicht in der Operation selbst begrüıdet waren, sonderı in den Veränderungen, die dırch dic Erkrankung im Organismus lıervorgerufeı waren.

Dies gilt jedoch nur von den Fällon, in welchen ein hochgradiger Meteorismus noch nicht besteht und in wclchen dic Desinvagination noch auszuführen ist. Ist crst ein lochgradiger Meteorismus vorhanden, so gestaltet sich der Fingriff wescntlich schwieriger und kann bei Paticnten, die, wie es dann gewöhulich ist, sich schon im Collaps befinden, viellcicht den üblen Ausgang: noch beschlcunigen.

Als ein Beispiel dieser ungïnstigen Fälle lasse ich einen vierten Fall folgen, den ich in der Privatpraxis opcrirt habe.

Fall 4. Knabe B., ein Jahr alt, erkranktc anı 30. April 1894, nach völligem Wohlbefinden, mit sehr heftigen Leibschmerzen. Die Schmerzen traten anfallswcise auf, waren so stark, dass das Kind sich im Bettchen aufbäumte und kaum zu halten war. Wiederholt trat Erbrechen cin, dem Stuhlgang, der am 30. April und 1. Mai noch kothig war, war Blut beigemischt. Vom 2. Mai ab wurden dic Schmerzanfülle inmer häufiger, so dass das Kind fast beständig vor Schmerzen schrie. Das Erbrechen liess nach, nachden das Kind nur noch Thee mit Cognac bekam. Stuhlgang scitden nur Blut und Schlcirn enthaltend. Collcge Dahmann diagnostirte eine Invagination und wandtc dic gebräuchlichen Mittcl ohnc Erfolg all.

Am 5. Mai Abends sah ich das Kind mit dem behandelnden Arzt. Es sah verfallen aus, der Gesichtsausdruck war ängstlich, der Puls klein und frequent, keine Temperatursteigerung. Der Leib war etwas anfgetricben, in der Gegend des Colon descendens fuhlte inan einen wurstförmigen Tumor, der nach oben bis zur Flexur reichte und sich nach unten ins kleine Becken erstreckte. Aus dem Anus ging blutiger Schleim ab. Mit dem in das Rectum eingeführten Finger fithlte naan ungcfähr $5 \mathrm{~cm}$ tiber dem Anus das invaginirte Darmstück, welches stark angeschwollen war. Nach den Erfahrungen von Dr. Harder schien es mir indicirt, sogleich die manuelle Reposition zu versuchen. Wahrend ich mit dem Zeigefinger der einen Hand vom Rectım aus das lntussusceptum nach oben zı schieben suchte. machte ich mit der anderen Hand die zwcekentsprechenden Manipulationen von den Bauchdecken aus, und es gelang mir so, dic Invagination soweit zu beseitigen, dass ich voun Rectmm aus nichts mehr fithlen konnte Ind dass auch von oben kein Tumor mehr nachzuweisen war. Ob die Invagination jedoch vollständig gehoben war, kann ich mit Sicherheit nicht angeben.

Die ersten Stunden der Nacht verlicfen recht ruhig, nach Mitternacht begannen jedoch die Schmerzen wieder nnd wurden gegen Morgen so heftig, dass Dr. Dahmann gerufen wurde. Derselbc fand dic Invagination wieder ebenso wie am Tage vorher und fuhrte die manuellc Reposition aus. Ich sah das Kind bald nachher und fand zwar vom Rectım aus keine Invagination mehr, dagcgen fïhlte ich jetzt ganz deıtlich cinen querverlaufenden wurstförmigen Tumor in der linken oberen Bauchgegend, der sicher als eine Invagination am Ende des Colon transversum anzusehen war. Das Kind war etwas matter als am T'age vorher, der Meteo- rismus hatte beträchtlich zugcnommen, man sah durch dic Baıchdecken hindurch die stark meteoristisch aufgeblähten Darmschlingen.

Unter diesen Umständen glaubte ich die Operation nicht weiter hinausschioben zu sollen und schritt zur Laparotomie. Ungefähr fingerlanger Bauchschnitt, zur Hälfte oberhalb, zur Hälfte unterhalb des Nabels licgend und diesen an der linken Seite umkreisend. Nach Eröff̈nung derBauchhøhle quellen sofort stark injicirte und zum Theil ad maximunı erweiterte Dünndarnıschlingen vor, die nur ınit Mühe reponirt und durch cine Compressc zuriickgehalten werdcn. Bei dem Versuche, die invaginirte Darmpartie hervorzuzichen, traten wieder reichlich Darmschlingen aus der Banchhöhle herans, die cinstweilcn mit eincr feuchten Compresse bedeckt wurden. Die invaginirte Stelle sass an der Flexura coli, war ungefähr fingerlang und liess sich leicht durch Zug und Druck desinvaginiren. Der Darm war an der Stelle stark geröthet, doch zcigten sich, nachdem er auseinandergczogen war, nirgends stärkcrc Ernährungsstörungen, nur an ciner Stelle fand sich ein grosser Blutaustritt nnter der Serosa. Der Darm wurde dann wieder reponirt, anch dic Dïnndarnıschlingen wurden reponirt, dahei entstand an einer selır stark crweiterten Düumdarmschlinge ein querer Einriss durch die Serosa. Dann wurde die Bauchhöhle schnell durch die Naht geschlossen. Der Puls war an Ende der Operation sehr klein. Das Kind war nur in halber Narkose gehalten worden. Nach der Ocgeration wimmerte das Kind schr, lag meist still, hatte sehr lebhaften Durst. Erbrechen trat nicht cin, dagegen wurde dic Respiration imnıcr oberflächlicher, der Puls immer kleiner. Acht Stınden nach der Operation Exitus.

Das Kind wurde am siebentell Tage nach Boginn der Elkrankung bei selıon bestehcudem starken Metcorismus und im collabirten Zustande operirt. Jer Meteorismus erscliwerte dic Opcration sclır, dic aufgeblähten Dünndarmschlingcı vorlegten den Weg zulr Invagination und inussten aus der Buchhöhlc ausgepackt werden. Dic Zurïicklagerung der Darmsclılingen in die Baıchhölıle gelanı nur mit grossel' Schwierigkeit. Dur'ch diescs alles wurde dic Operation bei dem klcinen Kinde zu einer schr eingr'eifenden, und es ist nicht zll verwundern, dass das Kind sieh aus dem Collaps nicht wieder erholte. Dass sclbst ganz kleine Kinder unter günstigen Unständen dic Opcration iiber'leben, beweist die Statistik von Wichmann, nach welcher von elf Kindern unter einem Jalır, die an er'sten Krankheitstage operirt wurden, sechs gelıeilt wurden.

Wic aus der an Fingang der Arbeit angefülırten Statistik lıervorgeht, ist kein einziger Patient bis zu zehn Jahren, bei welchem die Desinvagination nicht gelang, gehcilt worden. Aber anch bei älteren Individucn sind dic Chancen der Heilung dann in acuten Fällen nur geringe. Dor Fingriff, der, wenn die Desinvagination niclıt möglich war, gcwölınlich ausgefiilırt wurde, dic Rescetion der ganzen Invagination, ist cin zu sclıwerer Fingriff, wenigstens im acuten Stadium der Erkrankung, als dass vicle Patienten ihn ïberleben sollten. ${ }^{1}$ ) Man wird deshalb gut thun, dic Versuche, die Desinvagination herbeizuführen, nicht zu bald aufzugeben. Es ist sicher gerechtfertigt, wenn $\mathrm{Scun}$ empfichlt, in schwierigen Fällen dic etwa schon bestelıenden Verwachsungen stumpf oder auch mit Scheereuschlägen zu löscn. Auch die anderen Ratlıschlägc, dic Senn für solche Fälle giebt, sind zll beherzigen. Man soll das Intussusceptum du'ch Schwammdruck compriniren, un das Oedem, das häufig die Reposition hindert, zu bescitigen, und man soll eventuell die Manipulationen dırch Einblasen von Luft vom Mastdarm aus unterstützcn. Dass selbst nach sehr langem Bestelıcn der Invagination die Desinvagintation noch möglich ist, bewciscn die Fälle von Rydygicrón ${ }^{2}$ ), dem es in zwei Fällen noch nach sechs-, resp. achtinonatlichem Bestehen der Invagination gelang, die Invagination zu löseı. Gelingt es noch, die Invagination zu lösen, zeigt sich der Darm aber dann an einer Stclle gangränös oder der Gangrän verdächtig, so wird man am besten thun, den betreffenden Darmabschnitt cxtrapcritoneal zu lagern und erst später dic Darnresection auszuführen, wie es bercits in mchrercn Fällen, unter anderen von Linde man $n^{3}$ ), geschehen ist.

1) Nach Abschluss dieser Arbeit habe ich einen Fall operirt. der hierhin gehört und das oben Gesagte illıstrirt. Bei einem 72 jährigen Kapitän. der scit drei Tagen alle Erscheinungen eines completen Darmverschlusses darbot, fand sich bei der Untersuchung in Narkose cin wurstförmiger Tumor in der Ilcococealgegend. Bci der Laparotomie zeigte sich eine Invagination des unteren 'Theiles des Ileums und des Coecums in das Colon asceudens. Die Einkleummung am Halse der Invagination war eine ansserordentlich scharfe, das Intussusceptum war stark geschwollen, auch die Scheide war dunkelblauroth injicirt und riss bei dem Versucl der Desinvagination an mehreren Stcllen oberflächlich eill. In der Bauchhöhle fand sich blıtige Flüssigkeit, dic Dünndarmschlingen waren stark injicirt und hochgradig ansgedehnt. Es wurde die Resection der Invagination ausgefiihrt, dic beiden Darmenden wurden dirch die Naht g'eschlossen und eine seitliche Enteroanastomosc angelegt. Der Patient starb nach 24 Stunden. Dic Section crgab, dass die Naht völlig gehalten hatte, in der Banchhöhlc fand sich wenig blutige Flïssigkeit, kein Eiter, dic Dünndarmschlingen waren stark injicirt und ansgedehnt.

2) Majewski, ref. Centralblatt fiir Chirurgie 1895, S. 148.

3) Berliner' klin. Wochenschrift 1892, No. 23. 
Ist jedoch die Desinvagination unmöglich, so wird man auch nur bei kleinen Invaginationen und bei relativ gutem Kräftezustand des Patienten zur Resection schreiten, ist der Patient aber bereits so collabirt, dass er voraussichtlich die Resection nicht mehr aushält, so wird man die viel schneller auszuführende Enteroanastomose vornehmen. Diese wird man dann zweckmässig zugleich mit der Exstirpation des Intussusceptum von einem Längsschnitte der Scheide aus combiniren, wie es von $\mathrm{Bier}^{1}$ ) und Senn angegeben worden ist. Dieses letztere Verfahren, Exstirpation des Intussusceptums und Enteroanastomose, wird auch bei allen sehr grossen Invaginationen das einzige sein, welches Aussicht auf Erfolg bietet.

Die Enterostomie weist bei einer sehr grossen Anzahl von Misserfolgen nur einen einzigen Erfolg auf, sie wird daher auf die Fälle zu beschränken sein, in wclchen der Zustand des Patienten die oben angeführten radicaleren Eingriffe verbietet.

In chronischen Fällen sind die Resultate, die mit der Resection erzielt worden sind, relativ günstige, die Operation ist in diesen Fällen nicht so schwierig, da dann gewöhnlich Ileuserscheinungen nicht mehr bestehen, und übertrifft wohl an Schwierigkeit nicht cine Darmresection wegen Tumors. Ich hatte im Jahre $1891 \mathrm{Ge}-$ legenheit, eine chronische Invagination im Bereiche des Colon descendens zu reseciren, welche schon seit zwei Jahren bestand. Die Operation war recht inühsam und langdauernd, weshalb ich nicht die Darmnaht ausführte, sondern einen Anus praeternaturalis anlegtc. Das Befinden des Patienten war ein gutes bis zum nächsten Morgen, als sich plötzlich eine vollständige Hemiplegie einstellte, der der Patient an demselben Tage erlag. Die Section crgab eine Apoplexia cerebri als Todesursache. 